\title{
The Eye with Ears - A Symbol-Based Aid in Medical Teaching
}

\author{
Alexander Salava
}

\section{ABSTRACT}

Visual clinical examination forms the diagnostic basis in skin-related conditions and is an essential element in medical teaching. The author describes a symbol-based aid to explain the dichotomy of factual observed findings with the information based on medical history: The Eye with Ears. It represents the two most relevant aspects in clinical examination of skinrelated conditions and helps to clarify the contrast between the actual observation with information based on the context or past medical history. With the described aid, the author believes to have improved the quality of the teaching clinical examination and that it has functioned as a practical help for medical students' and residents' professional development.

Keywords: Clinical examination, dermatology, medical education, skinrelated conditions, visual observation skills.

Submitted: July 9, 2021

Published: September 21, 2021

ISSN: 2593-8339

DOI: $10.24018 /$ ejmed.2021.3.5.977

Alexander Salava*

University of Helsinki, Finland.

Department of Dermatology and Allergology, Helsinki University Hospital, Finland.

(e-mail: alexander.salava@helsinki.fi)

*Corresponding Author

\section{INTRODUCTION}

Visual clinical examination forms the diagnostic basis when dealing with skin-related conditions and comprises essential elements that need to be learned in medical education [1]. As skin related conditions are frequent causes of consultations in primary care, dermatology not only reflects a small subspecialty; it should be regarded as a key curricular component in clinical examination [2]. The "skin approach" is based on visual observation; the physician looks for signs and symptoms (morphology), recognizes patterns and gathers information (visual inventory) [3]. However, the importance of all findings needs to be weighted and appreciated in light of the patient history, context and dynamics of the specific problem [4]. The process has multiple visual and cognitive levels, but for novices the difference of observation and inference is pivotal to understand for a similar observation, the diagnostic meaning can vary considerably (differential diagnoses, alarm signs etc.) [5]. Thus, the patient with a skin-related condition needs to be examined by integrating the observable skin changes (factual) with other information, which is mainly acquired by listening (patient history).

Visual observational skills have undergone a renaissance in medicine in recent years [6], [7]. Many institutions of higher education accept the training of visual observation as an important content of the curriculum; often linked to the dermatology course [8], [9]. In undergraduate teaching there have been some publications on the role of art in improving observational skills and on the use of perceptual learning modules [10]. It has become clear that visual diagnostic skills are strongly based on conceptual learning (as opposed to declarative and procedural learning), can be learned, and are not simply based on habituation or experience [11]. Nevertheless, teaching clinical examination on patients with skin-related conditions remains a challenge and is still deficient of a simple systematic framework.

\section{TEACHING INNOVATION}

The author has been teaching skin-related clinical examination to undergraduate medical students since 2015 at the University of Helsinki, Finland. The teachings have included voluntary 2 -week courses for $4^{\text {th }}$ year medical students with practical training of observational skills in an art museum and bedside clinical examination of patients. In addition, teaching of clinical examination (bedside and in small groups) has been an integral part of the obligatory course of dermatology for $5^{\text {th }}$ year medical students.

During the teachings, the author has used a symbol-based aid to explain the dichotomy of factual observed information with the medical history-based information: The Eye with Ears. With the described aid, the author believes to have improved the quality of the teaching clinical observation and that it has functioned as a practical aid for the professional development of medical students and residents.

The Eye with Ears symbol consists of a central human eye, with two small human ears attached on the upper sides of the eye. It represents the two most relevant aspects in clinical examination of skin-related conditions and helps to clarify the contrast of observed information with the information based on the context and past medical history. Here, the eye represents the visual information, which is observed during clinical examination: skin changes and morphology, pattern recognition and localization. On the contrary, the ears symbolize contextual information, which needs to be acquired via different channels, mainly by listening, contextualizing, and obtaining the past medical history.

With the symbolic presentation, the aim is to concretize the fact that similar observations can lead to multiple 
conclusions and need to be interpreted in the light of the entire clinical situation. The ears are also used to represent important aspects that are hidden from the eyes of the physician but need nevertheless to be considered when interpreting findings: imitating or masquerading diseases, differential diagnoses, visual and cognitive bias etc.

\section{CONCLUSION}

The Eye with Ears symbol can be used on lecture slides or attached to patient cases, in bedside teaching or during group works as a reminder and memory aid to point out the essential difference between visual information (what we see with the eye), contextual information (what we hear in the patient's past medical history) and inference. In the experience of the author the greatest educational advantage is achieved when the symbol is showed repeatedly in intervals (e.g., during presentations of complicated patient cases) or highlighted when a specific problem of clinical examination is discussed (difficult or large amount of findings, conflicting clinical pictures, discrepancy of skin changes with other clinical information).

By repeatedly presenting the Eye and Ears symbol the teacher can help to shift the focus of students to the "skin approach" and the difference of observation and inference is visually pointed out. This may be of practical importance during teaching of clinical examination in skin-related conditions as the great challenge in visual observational skills is the lack of concrete structure in the actual process of examination. Furthermore, the described symbol helps students to memorize the most important pitfall of visual observation, which frequently occurs to novices during clinical examination of patients with skin-related conditions.

\section{LESSONS FOR PRACTICE}

- Visual clinical examination is an essential element in the practice of medicine and needs to be taught in undergraduate education.

- The author describes a symbol-based aid to explain the dichotomy of factual observed information with information based on medical history: The Eye with Ears.

- The aid may improve the quality of the teaching clinical examination and function as a practical help for medical students' and residents' professional development.

\section{ACKNOWLEDGMENT}

The author reports no conflict of interest. There were no funding resources that supported this work.

\section{REFERENCES}

[1] Braverman IM. To see or not to see: how visual training can improve observational skills. Clin Dermatol. 2011;29(3):343-346.

[2] Salava A, Oker-Blom A, Remitz A. The spectrum of skin-related conditions in primary care during 2015-2019-A Finnish nationwide database study. Skin Health and Disease. 2021;e253.

[3] Boudreau JD, Cassell EJ, Fuks A. Preparing medical students to become skilled at clinical observation. Med Teach. 2008;30(910):857-862.
[4] He B, Prasad S, Higashi RT, Goff HW. The art of observation: a qualitative analysis of medical students' experiences. BMC Med Educ. 2019;26;19(1):234.

[5] Miller A, Grohe M, Khoshbin S, Katz JT. From the galleries to the clinic: applying art museum lessons to patient care. J Med Humanit. 2013;34(4):433-438.

[6] Lyon P, Letschka P, Ainsworth T, Haq I. An exploratory study of the potential learning benefits for medical students in collaborative drawing: creativity, reflection and 'critical looking'. BMC Med Educ. 2013;17;13:86.

[7] Guegan S, Streichen O, Soria A. Literature review of perceptual learning modules in medical education: What can we conclude regarding dermatology? Ann Dermatol Venereol. 2021;148:16-22.

[8] Cipriano SD, Dybbro E, Boscardin CK, Shinkai K, Berger TG. Online learning in a dermatology clerkship: piloting the new American Academy of Dermatology Medical Student Core Curriculum. $J$ Am Acad Dermatol. 2013;69(2):267-272.

[9] Choi AW, Xu RS, Jacob S, Dulmage BO, Colavincenzo ML, Robinson JK, Xu S. Visual perception training: a prospective cohort trial of a novel, technology-based method to teach melanoma recognition. Postgrad Med J. 2019;95(1124):350-352.

[10] Miller A, Grohe M, Khoshbin S, Katz JT. From the galleries to the clinic: applying art museum lessons to patient care. J Med Humanit. 2013;34(4):433-438.

[11] Kellman PJ, Krasne S. Accelerating expertise: Perceptual and adaptive learning technology in medical learning. Med Teach. 2018;40(8):797-802.

Alexander Salava, M.D., Ph.D., is Clinical Lecturer of Dermatology at the University of Helsinki, Finland. His main pedagogical interests are clinical observation and examination skills in dermatology and perceptual learning. Dr. Salava has had the possibility to acquire a broad clinical experience as a specialist in dermatology at the Helsinki University Hospital and abroad and has been actively involved in medical research. 